\title{
Bevacizumab Modulation of the Interaction Between the MCF-7 Cell Line and the Chick Embryo Chorioallantoic Membrane
}

\author{
ŞERBAN COMŞA ${ }^{1}$, ROXANA POPESCU ${ }^{2}$, ŞTEFANA AVRAM ${ }^{1}$, \\ RALUCA AMALIA CEAUȘU ${ }^{1}$, ANCA MARIA CIMPEAN ${ }^{1}$ and MARIUS RAICA ${ }^{1}$ \\ ${ }^{1}$ Department of Histology, Angiogenesis Research Center and ${ }^{2}$ Department of Cell and Molecular Biology, \\ Victor Babeş University of Medicine and Pharmacy, Timişoara, Romania
}

\begin{abstract}
Aim: To evaluate the interaction between $M C F$ 7 breast cancer cells and the chick embryo chorioallantoic membrane (CAM) and the ability of bevacizumab to modulate this process. Materials and Methods: We implanted MCF-7 cells onto CAM and repeatedly added bevacizumab to a subset of eggs. We then evaluated the morphological and immunohistochemical profiles of CAM and MCF-7. Results: MCF-7 cells entered the mesoderm and stimulated the mesenchymal cells to acquire vasculogenic and myofibroblastoid features. MCF-7 cells developed an estrogen receptor-, progesterone receptor-, p53- and Ki67negative status and entered the epithelial-mesenchymal transition. Bevacizumab down-regulated the expression of $B$ cell lymphoma 2 protein (BCL-2), vascular endothelial growth factor (VEGF) and E-cadherin in MCF-7 and inhibited vasculogenesis. Conclusion: MCF-7 cells turn the mesoderm of CAM into a surrogate tumor stroma. CAM induces a triple-negative, non-proliferative but still antiapoptotic status in MCF-7 cells. Although antivasculogenic, bevacizumab stimulates MCF-7 cells to acquire a more aggressive status.
\end{abstract}

In vivo angiogenesis assays, such as those performed in the chick embryo chorioallantoic membrane (CAM), have made important progress in elucidating the mechanisms of action of several angiogenic factors and inhibitors (1). At the moment, CAM is widely utilized as an in vivo system to study angiogenesis and anti-angiogenesis (2). It has also long

This article is freely accessible online.

Correspondence to: Anca Maria Cîmpean, Victor Babeş University of Medicine and Pharmacy, Department of Histology, Piaţa Eftimie Murgu nr. 2, 300041, Timişoara, Timiş, Romania. Tel: +40 720060955 , Fax: +40 256490626, e-mail: ancacimpean1972@yahoo.com

Key Words: Angiogenesis, bevacizumab, breast cancer, chorioallantoic membrane, mesenchymal stem cells. been a favored system for the study of tumor angiogenesis and metastasis, because at this stage, the chick's immunocompetence system is not fully developed and the conditions for rejection have not been established (1). This in vivo model is simple, inexpensive, experimentally amenable, as it readily accepts and supports xenogenic transplants (3).

Chick embryo model systems offer a number of unique advantages for studying the complex, multistep process of tumor cell metastasis. Different chick embryo model systems allow comprehensive analysis of specific stages and aspects of cancer cell dissemination, such as tumor cell intravasation in the spontaneous metastasis model, tumor cell colonization in the experimental metastasis model, and tumor-induced angiogenesis in the collagen implant model (4).

MCF-7 is a commonly used breast cancer cell line, being propagated for many years by multiple research groups (5). Established in 1973 by Soule and colleagues at the Michigan Cancer Foundation, MCF-7 cells were isolated from the pleural effusion of a 69-year-old woman with metastatic disease (6). Since its establishment, MCF-7 has become the model of estrogen receptor (ER)-positive breast cancer (7). In a previous review, we summarized the molecular profile of the MCF-7 cell line, concluding that it is ER-and progesterone receptor (PR)-positive, belongs to the luminal A molecular subtype, is poorly aggressive and non-invasive, with low metastatic and angiogenic potential (8).

The controversial vascular endothelial growth factor (VEGF)-specific angiogenesis inhibitor, Avastin (bevacizumab), is currently approved in Europe for human epidermal growth factor receptor 2 (HER2)-negative metastatic breast cancer in combination with paclitaxel or capecitabine (9), but its breast cancer indication was withdrawn in 2011 by the US Food and Drug Administration after concluding that the drug had not been proven to be safe and effective for the treatment of breast cancer (10).

The aim of our study was to evaluate the effects of MCF-7 breast cancer cell implantation onto CAM, through changes in both the morphological and immunohistochemical aspects 
regarding the MCF-7 cells and CAM. In addition, we aimed to analyze the ability of bevacizumab to influence these changes.

\section{Materials and Methods}

MCF-7 cell culture. MCF-7 cells (CLS Cell Lines Service GmbH, Eppelheim, Germany) were cultured at a density of $1 \times 10^{6}$ cells/T75 plate in Dulbecco's modified Eagle's medium (Life Technologies Europe BV, Paisley, Scotland) containing $10 \%$ fetal bovine serum (Life Technologies Europe BV, Bleiswijk, the Netherlands) and 1\% mixture of penicillin and streptomycin (LIife Technologies Europe $\mathrm{BV}$, Bleiswijk, the Netherlands), and incubated at $37^{\circ} \mathrm{C}$ in a humidified atmosphere containing $5 \% \quad \mathrm{CO}_{2}$. The medium was changed twice per week and cells were passaged once a week, at a subcultivation ratio of $1: 3$.

The CAM experimental model preparation. A total of 32 fertilized chicken eggs were incubated at $37^{\circ} \mathrm{C}$. On day 3 of incubation, a window in the eggshell was made and covered with Parafilm. The eggs were then re-incubated for another 7 days.

The implantation of MCF-7 cells onto CAM and bevacizumab treatment. On day 10 of incubation (day 1 of the experiment), a ring of silicone was placed onto the CAM surface of each egg under sterile conditions. The eggs were divided into three subsets: control: 4 eggs, subset 1: 14 eggs and subset 2: 14 eggs. MCF-7 growth medium $(5 \mu \mathrm{l})$ was added inside the rings of the control eggs, while $5 \mu \mathrm{l}$ of growth medium with a suspension of MCF-7 cells $\left(2 \times 10^{4}\right)$ was inserted inside the rings of the eggs of subsets 1 and 2 . In addition, each egg from subset 2 received $2 \mu \mathrm{l}$ of Avastin $(25 \mathrm{mg} / \mathrm{ml}$ bevacizumab) on days 1, 3 and 5 of the experiment (i.e. day 10, 12 and 14 of total incubation). On day 7 (day 16 of total incubation), the experiment was stopped through the in ovo instillation of $5 \mathrm{ml}$ of $4 \%$ formalin onto the surface of the CAM. During the incubation, the viability of the eggs was assessed daily, as a prerequisite to completing the experiment.

Morphological evaluation of the CAM. In order to perform the morphological evaluation, the CAM of each egg was detached and submerged for fixation in $4 \%$ buffered formalin, for 48 hours, thereafter placing the embedding in paraffin. Sections 5-mm-thick were then cut by using a microtome and hematoxylin and eosin staining was then performed.

Immunohistochemical evaluation of the CAM. Incubation for 15 minutes was carried out using the following Bond ready-to-use primary antibodies: ER, PR, HER2, cytokeratin 5 (CK5), CK18, $\alpha$ smooth muscle actin ( $\alpha$ SMA), E-cadherin, Ki67, p53, B-cell lymphoma protein (BCL2), $\beta$-catenin and vimentin from Leica Biosystems (Newcastle Ltd, Newcastle upon Tyne, UK) and VEGF from ReliaTech (Wolfenbüttel, Germany). Bond Polymer Refine Detection System (Leica)was used for visualization. The chromogen dihydrochloride-3,3-diamine-benzidine was applied for 10 minutes. In the case of $\beta$-catenin/vimentin double immunostaining, Bond Polymer Refine Red Detection System (Leica) was used as the second visualization system. Lille's hemotoxylin was used as counterstain for 5 minutes. The entire procedure was performed using a Leica Bond-Max autostainer (Leica Biosystems).
The immunoexpression was evaluated as follows: nuclear expression for ER, PR, Ki67 and p53; cytoplasmic expression for CK5, CK18, $\alpha$ SMA, vimentin, $\beta$-catenin and BCL2; membranous for HER2 and E-cadherin. Absence of the immunostaining was considered as negative (-), while the presence of immunohistochemical reaction was graded according to the interpretation protocol given by the manufacturer as low (+), or moderately $(++)$, or highly $(+++)$ positive.

\section{Results}

A total of 22 eggs survived the experiment: three in the control group, 10 in subset 1 and nine in subset 2 . The CAM of these eggs was evaluated in order to assess the morphology and the immunohistochemical profile.

The morphological evaluation of CAM. In subset 1 (MCF-7 cells alone), the morphological evaluation revealed a high number of tumor cells on the CAM with a frequent tendency for necrosis. The tumor cells adhered to each other and to the chorionic epithelium. They also managed to cross this epithelium and enter the mesoderm. Inside this layer, the MCF-7 cells developed luminal structures resembling mammary acini or ducts. The tumor cells also entered the vessels of the mesoderm and changed their own morphology, through the appearance of cell elongations connecting them with each other and with the endothelium (Figure 1A). The presence of MCF-7 cells stimulated the mesenchymal cells from the mesoderm to acquire myofibroblastic-like features, especially in the perivascular areas. MCF-7 also induced an intense vasculogenic reaction in the peritumoral areas in the mesoderm, by stimulating the mesenchymal cells to organize into capillary-like structures (Figure 1B). The mechanism of vasculogenesis identified in these areas was cell hollowing, through vacuole coalescence. Inside the newly formed capillary-like structures, hematopoietic progenitors were identified, indicating that vasculogenesis and hematopoiesis were simultaneous and co-localized processes.

In subset 2 (MCF-7 plus bevacizumab), in the presence of bevacizumab, there was a lower number of tumor cells and no tendency for necrosis compared to subset 1 . In addition, the evaluation of the mesoderm showed reduced myofibroblastic and vasculogenic reactions.

In the control subset, no myofibroblastoid or vasculogenic reactions were identified.

The immunohistochemical profile of MCF-7 and CAM. After implantation onto CAM, MCF-7 cells lost their expression of ER, PR and Ki67 and p53, developed a moderately positive VEGF status, associated with a low expression of BCL2, acquired a phenotype suggestive of the epithelialmesenchymal transition: vimentin-positive, heterogeneous as distribution and intensity for $\beta$-catenin and CK18 expression (Table I, Figure 1C). 

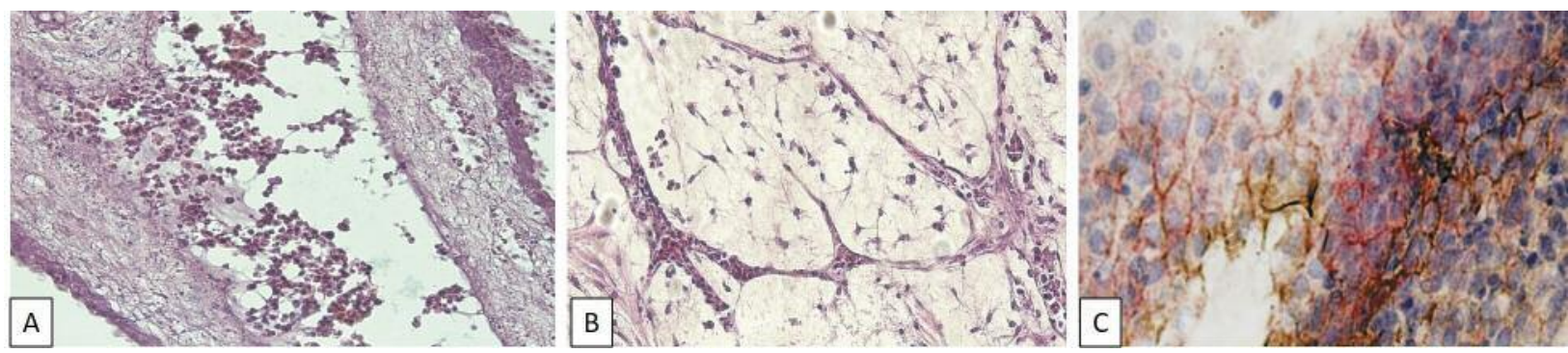

Figure 1. A: MCF-7 cells enter the mesodermal vessels and develop cell elongations connecting them with each other and with endothelium cell elongations. Hematoxylin-eosin staining, magnification $\times 400$. B: Chick embryo chorioallantoic membrane (CAM) mesoderm after MCF-7 cell implantation. Vasculogenic reaction: cell hollowing and vacuole coalescence; simultaneous and co-localized vasculogenesis and hematopoiesis. Hematoxylin-eosin staining, magnification $\times 400$. MCF-7 cells on CAM. C: Epithelial-mesenchymal transition is demonstrated by the loss of Ecadherin expression (brown) and the low expression of vimentin (red). Immunohistochemistry, magnification $\times 400$.

Table I. The immunohistochemical profile of MCF-7cells cultured on chick embryo chorioallantoic membrane (CAM). Comparison with the profile of the MCF-7 parental line, as described in literature. The expression of the parental line markers was evaluated either immunohistochemically (including Allred score), by western blot (WB) or by enzyme-linked immunosorbent assay (ELISA).

\begin{tabular}{lcccc}
\hline Marker & Protein & MCF-7 & MCF-7/CAM \\
\cline { 3 - 5 } & & Parental line (Ref) & Subset 1 & Subset 2 (+Avastin) \\
\hline ER & Estrogen receptor & 6 - Allred (11) & - & - \\
PR & Progesterone receptor & $6-$ Allred (11) & - & - \\
HER2 & Human epidermal growth factor receptor 2 & $-/+(11)$ & - & - \\
VEGF & Vascular endothelial growth factor & Low (ELISA) (12) & ++ & + \\
p53 & Tumor protein p53 & $60 \%(13)$ & - & - \\
Ki67 & Ki67 protein & $40 \%(13)$ & - & - \\
BCL2 & B-Cell lymphoma 2 protein & $60 \%(13)$ & + & + \\
E-Cadherin & Epithelial-cadherin & $90 \%(13)$ & - \\
CK5 & Cytokeratin 5 & Negative (11) & + & + \\
$\beta$-Catenin & $\beta$-Catenin & Positive (WB) (14) & $++/-$ & $+/-$ \\
Vimentin & Vimentin & Negative (WB) (14) & $+/-$ & $+/-$ \\
CK18 & Keratin 18 & Positive (WB) (14) & +- \\
\hline
\end{tabular}

aSMA expression was positive in all zones with morphological myofibroblastic reaction but also in the mesenchymal tissue of the mesodermal layer, especially in the perivascular areas. When exposed to bevacizumab, MCF7 cells lost the expression of BCL2, suffered downregulation of VEGF and E-cadherin expression, with the consequent inhibition of vasculogenesis. In the control subset, ER, PR, HER2, CK5, CK18, VEGF, E-cadherin, $\beta$ catenin, vimentin, Ki67, p53 and BCL2 were not expressed.

\section{Discussion}

Our work provides a consistent analysis of the interaction between MCF-7 cells and CAM through the description of their morphological and molecular changes, allowing for establishment of an in vivo experimental model involving
MCF-7 breast cancer cells and CAM. Furthermore, using this established experimental model, we evaluated the ability of bevacizumab to modulate the tumorigenic processes in MCF7 cells developing on CAM.

On CAM, the tumor cells seem to have had a more favorable environment for survival in the absence of bevacizumab, such that it is clear that the drug led to the apoptosis of a significant number of tumor cells. Although it is well known that the CAM represents an appropriate medium for tumor cell survival, as long as the cells rest on the chorionic epithelium and do not enter the mesoderm, the nutrient supply is limited and cannot support more than a limited number of cells. This is why, in the absence of bevacizumab, the high number of tumor cells residing onto the chorionic epithelium presented a great percentage of necrosis. 
We reported that the tumor cells managed to cross the chorionic epithelium and entered not only the mesoderm, but also its vessels. This behavior reflects the aggressiveness and invasiveness of this cell line in this model, although the parental cell line is poorly aggressive and non-invasive, with a low metastatic potential (15). The CAM environment is responsible for this behavioral switch and, as mesenchymal stem cells seem to be able to stimulate MCF-7 migration (16), mesenchymal cells from the mesoderm might be the key factors in this process. MCF-7 migration might be related to the observed decrease in E-cadherin, as this molecule is the major protein mediating MCF-7 cell-cell adhesion in spheroids (16).

The behavior of neoplastic cells cannot be understood without taking into account their active and reciprocal dialogue with their microenviroment (17). It has been demonstrated that myofibroblasts are a major component of the breast cancer mass and key effectors of tumor progression (18). In addition, MCF-7-derived exosomes have proven ability to convert mesenchymal stem cells within tumor stroma into tumorassociated myofibroblasts (19). Our results therefore clarify these issues in the current experimental model, showing that although the MCF-7 cells are human and the mesenchymal cells of chick origin, the tumor cells have a strong influence on the mesenchymal cells of the mesoderm, as they stimulate them to acquire a myofibroblastoid phenotype. Interestingly, $\alpha$ SMA expression was not limited to cells with morphological myofibroblastic features, but was also found in mesenchymal cells with classical morphology, suggesting that this marker is activated early in the phenotype-changing process, even before the initiation of the morphological changes.

Although MCF-7 cells usually have a poor angiogenic potential (20) and secrete immunodetectable but low levels of VEGFA (12), our results demonstrated that MCF-7 cells develop a highly vasculogenic potential on CAM and present a moderate immunohistochemical expression of VEGF. We previously showed that mesenchymal stem cells have a clear tendency to organize into clusters and to form capillary-like structures in Matrigel, in the presence of MCF-7 cells (21), but it is interesting to note that similar structures were identified in the current in vivo model, through the stimulation of MCF-7 cells and the involvement of the mesenchymal cells from the mesoderm. The mechanism of vasculogenesis we identified was cell hollowing, through vacuole coalescence. This model has been proposed by important names in the field, although, lately, cord hollowing has been considered more likely to be the common mechanism of vascular lumen formation in the majority of blood vessels (22).

It is important to mention that Ribatti et al. clearly noted that immature blood vessels (lacking a complete basal lamina and smooth muscle cells), scattered in the mesoderm, grow very rapidly until day 8 of incubation (1), while our vasculogenic reactions were identified on day 17 of incubation. We propose that MCF-7 cells somehow reset the evolution of the CAM, setting it into an early, immature stage.

Witkiewicz et al. demonstrated that not only vessels but also blood formed de novo at the location of growing tissue, not just in bone marrow and spleen (23). We confirmed this process in the MCF-7/CAM model, by demonstrating that inside the newly formed capillary-like structures, hematopoietic progenitors were identified.

After implantation onto CAM, MCF-7 cells changed their molecular profile and became triple-negative, suggesting the acquisition of a more aggressive status. Selection of triplenegative sublines from the ER-positive MCF-7 cell line has been previously achieved using the anti-estrogen fulvestrant, a complete ER antagonist (24). It remains to be studied whether the CAM environment exerts inhibitory ER effects, through a specific factor.

Generally, the more aggressive types of breast tumors, which are hormone-independent, are BCL2-negative. On the other hand, an inverse relationship usually exists between p53 and BCL2 status (25). Although the tumor cells we implanted onto CAM became ER- and PR-negative, BCL2 expression decreased and was lost only in the presence of bevacizumab. Regarding the p53 and BCL2 status, there was no inverse relationship, as reported before, possibly because of an unidentified overexpression of mutant p53 in the MCF-7 cells which could down-regulate BCL2 (25), as our immunohistochemical evaluation was focused on wild-type p53.

Among other features suggestive of a more aggressive status, the MCF-7 cells implanted onto CAM also entered the process of epithelial-mesenchymal transition. It is important to underline that the mechanisms through which MCF-7 cells become more aggressive on CAM might be related to each other. A good example is the confirmation from a previous study that in luminal invasive breast cancer, the epithelial-mesenchymal transition and the loss of ER $\alpha$ expression were linked (26).

Although efficient in reducing VEGF expression and the vasculogenic process, bevacizumab stimulated the process of epithelial-mesenchymal transition in our study, as it augmented the E-cadherin decrease in MCF-7 cells. Others have shown that bevacizumab activates alternate angiogenesis pathways, such as through pleiotrophin upregulation. Pleiotrophin functions as an angiogenic factor and promotes remodeling of the tumor microenvironment, as well as epithelial-mesenchymal transition (27).

\section{Conclusion}

MCF-7 cells recruit mesenchymal cells and most probably induce their differentiation through myofibroblast-like lineage or cells with a high vasculogenic potential, turning the mesoderm of CAM into a surrogate tumor stroma. The CAM environment changes the molecular profile of MCF-7 
cells and induces an aggressive, non-proliferative, but still anti-apoptotic state. CAM stimulates the epithelialmesenchymal transition in MCF-7 cells; although antiangiogenic, bevacizumab might stimulate this process, together with the loss of BCL2 expression, suggesting the acquisition of a more aggressive status.

\section{Acknowledgements}

This work was supported by the P II - C3 - TC - 2015 of "Victor Babeş" University of Medicine and Pharmacy, Timişoara, Romania.

\section{References}

1 Ribatti D, Nico B, Vacca A, Roncali L, Burri PH and Djonov V: Chorioallantoic membrane capillary bed: A useful target for studying angiogenesis and anti-angiogenesis in vivo. Anat Rec 264: 317-324, 2001.

2 Ribatti D, Vacca A, Roncali L and Dammacco F: The chick embryo chorioallantoic membrane as a model for in vivo research on angiogenesis. Int J Dev Biol 1197: 1189-1197, 1996.

3 Quigley JP and Armstrong PB: Tumor cell intravasation alu-cidated: the chick embryo opens the window. Cell 94: 281-284, 1998

4 Auerbach R, Lewis R, Shinners B, Kubai L and Akhtar N: Angiogenesis assays: a critical overview. Clin Chem 40: 32-40, 2003.

5 Baguley BC and Leung E: Heterogeneity of phenotype in breast cancer cell lines. In: Breast Cancer - Carcinogenesis, Cell Growth and Signalling Pathways. Gunduz M and Gunduz E (eds.). Rijeka, InTech, pp. 245-256, 2011.

6 Soule HD, Vazguez J, Long A, Albert S and Brennan M: A human cell line from a pleural effusion derived from a breast carcinoma. J Natl Cancer Inst 51: 1409-1416, 1973.

7 Keen JC: Breast cancer cell line development and authentication. In: Breast Cancer - Focusing Tumor Microenvironment, Stem Cells and Metastasis. Gunduz M, Gunduz E (eds.) Rijeka, InTech, pp. 3-20, 2011.

8 Comşa Ş, Cîmpean AM and Raica M: The Story of MCF-7 Breast Cancer Cell Line: 40 years of Experience in Research. Anticancer Res 35: 3147-3154, 2015.

9 Prat A, Pineda E, Adamo B, Galvan P, Fernandez A, Gaba L, Díez M, Viladot M, Arance A and Munoz M: Clinical implications of the intrinsic molecular subtypes of breast cancer. Breast 24(Suppl 2): S26-35 2015.

10 Sasich LD and Sukkari SR: The US FDAs withdrawal of the breast cancer indication for Avastin (bevacizumab). Saudi Pharm J 20: 381-385, 2012.

11 Subik K, Lee JF, Baxter L, Strzepek T, Costello D, Crowley P, Xing L, Hung M-C, Bonfiglio T, Hicks DG and Tang P: The expression patterns of ER, PR, HER2, CK5/6, EGFR, KI-67 and AR by immunohistochemical analysis in breast cancer cell lines. Breast Cancer Basic Clin Res 4: 35-41, 2010.

12 Timoshenko A V, Chakraborty C, Wagner GF and Lala PK: COX2-mediated stimulation of the lymphangiogenic factor VEGF-C in human breast cancer. Br J Cancer 94: 1154-1163, 2006.

13 Lukyanova NY, Rusetskya N V, Tregubova NA and Chekhun VF: Molecular profile and cell cycle in MCF-7 cells resistant to cisplatin and doxorubicin. Exp Oncol 31: 87-91, 2009.
14 D’Anselmi F, Masiello MG, Cucina A, Proietti S, Dinicola S, Pasqualato A, Ricci G, Dobrowolny G, Catizone A, Palombo A, Bizzarri M: Microenvironment promotes tumor cell reprogramming in human breast cancer cell lines. PLoS One 8: e83770, 2013.

15 Gest C, Joimel U, Huang L, Pritchard LL, Petit A, Dulong C, Buquet C, Hu CQ, Mirshahi P, Laurent M, Fauvel-Lafève F, Cazin L, Vannier JP, Lu H, Soria J, Li H, Varin R, Soria C: Rac3 induces a molecular pathway triggering breast cancer cell aggressiveness: differences in MDA-MB-231 and MCF-7 breast cancer cell lines. BMC Cancer 13: 63, 2013.

16 Dittmer A, Hohlfeld K, Lützkendorf J, Müller LP and Dittmer J: Human mesenchymal stem cells induce E-cadherin degradation in breast carcinoma spheroids by activating ADAM10. Cell Mol Life Sci 66: 3053-3065, 2009.

17 Wernert N: The multiple roles of tumour stroma. Virchows Arch 430: 433-443, 1997.

18 Mehner C and Radisky DC: Triggering the landslide: the tumorpromotional effects of myofibroblasts. Exp Cell Res 319(11): 1657-1662, 2013.

19 Cho JA, Park H, Lim EH and Lee KW: Exosomes from breast cancer cells can convert adipose tissue- derived mesenchymal stem cells into myofibroblast-like cells. Int J Oncol 40(1): 130138, 2012.

20 Aonuma M, Saeki Y, Akimoto T, Nakayama Y, Hattori C, Yoshitake Y, Nishikawa K, Shibuya M and Tanaka NG: Vascular endothelial growth factor overproduced by tumour cells acts predominantly as a potent angiogenic factor contributing to malignant progression. Int J Exp Pathol 80: 271-281, 1999.

21 Comşa S,, Ciuculescu F and Raica M: Mesenchymal stem celltumor cell cooperation in breast cancer vasculogenesis. Mol Med Rep 5: 1175-1180, 2012.

22 Lammert E and Axnick J: Vascular lumen formation. Cold Spring Harb Perspect Med 2: 1-10, 2012.

23 Witkiewicz H, Oh P and Schnitzer JE: Embryonal vasculature formation recapitulated in transgenic mammary tumor spheroids implanted pseudo-orthotopicly into mouse dorsal skin fold: the organoblasts concept. F1000Research 2: 8, 2013.

24 Leung E, Kim JE, Askarian-Amiri M, Finlay GJ and Baguley C: Evidence for the existence of triple-negative variants in the MCF-7 breast cancer cell population. Biomed Res Int 2014: 17, 2014.

25 Basu A and Haldar S: The relationship between Bcl2, Bax and p53: consequences for cell cycle progression and cell death. Mol Hum Reprod 4: 1099-1109, 1998.

26 Guttilla IK, Phoenix KN, Hong X, Tirnauer JS, Claffey KP and White BA: Prolonged mammosphere culture of MCF-7 cells induces an EMT and repression of the estrogen receptor by microRNAs. Breast Cancer Res Treat 132: 75-85, 2012.

27 Lynn KD, Roland CL and Brekken RA: VEGF and pleiotrophin modulate the immune profile of breast cancer. Cancers 2: 970988, 2010.
Received November 27, 2016

Revised January 23, 2017

Accepted January 24, 2017 